\title{
The Investigation on Mask and Plasticine Laser Shock Forming
}

\author{
Liyin $\mathrm{LI}^{1, \mathrm{a}^{*}}$, Xiao WANG ${ }^{1, \mathrm{~b}}$, Zongbao SHEN ${ }^{1, \mathrm{c}}$, Huixia $\mathrm{LIU}^{1, \mathrm{~d}}$ and Youjuan MA ${ }^{1, \mathrm{e}}$ \\ ${ }^{1}$ School of Mechanical Engineering, Jiangsu University, Zhengjiang, Jiangsu 212000 \\ allyliliyin@163.com ${ }^{1}$, ${ }_{w x} @$ ujs.edu.cn, ${ }^{\mathrm{c}} \mathrm{szb} @$ ujs.edu.cn, ${ }^{\mathrm{d}} \mathrm{lhx} @$ ujs.edu.cn, ${ }^{\mathrm{e}} \mathrm{myj} @$ ujs.edu.cn
}

\begin{abstract}
A novel technology called mask and plasticine laser shock forming (MAPLSF) was introduced to manufacture micro-features on the surface of pure copper foil with the thickness of $40 \mu \mathrm{m}$. In this process, the laser-generated shock wave and plasticine are used to replace the conventional rigid punch and die. The result showed that the shape of laser beam can be roughly manufactured on the surface of the workpiece and this mold-free laser shock forming process is a fast method to fabricate controlled micro-feature on the metallic foils. In this damage-free forming process, the workpicec can keep good surface, because the plasticine is softer than metal foils.
\end{abstract}

\section{Introduction}

With the constant improvement of macro manufacturing, people gradually begin to research in the micro field [1-2]. Micro parts made by sheet metal are widely used in the fields of communications, electronics, and micro-electromechanical systems. There are many forming methods such as cold plastic and micro bulk forming [3-4].

However tradition plastic forming methods have many disadvantages. As we all know, it is difficult and expensive to fabricate the micro-molds using common methods. Also, micro-features on different locations of the die included upper surface, channel side wall and bottom of the die will be replicated onto the specimen surface, which will change the surface roughness of work pieces [5]. Therefore, it is desirable to develop a mold-free manufacturing process that can produce micro-features on metal sheets or foils.

In recent years, laser shock forming-a new forming method that has the advantage of tool-free, high efficiency, and high precision has been fully studied and widely applied. Zhou et al [6] utilized laser shock waves to deform austenitic and ferrite stainless steel sheets with the thickness of $0.3 \mathrm{~mm}$ to $0.9 \mathrm{~mm}$. Cheng et al [7] used the technology of laser dynamic forming to deform copper foils with the thickness of $15 \mu \mathrm{m}$ into micro-mold with width ranging from 200 to $300 \mu \mathrm{m}$. Vollertsen et al [8] utilized the laser deep drawing technique to fabricate copper, aluminum, and stainless steel sheets with the thickness of $20 \mu \mathrm{m}$ into spherical cups of $1 \mathrm{~mm}$ height using a TEA-CO2 laser. Laser technology has gradually matured, and has been widely used in the forming area. Because of the maturity of the laser shock forming technology, some scientists use laser as a punch to research forming without

\footnotetext{
*Corresponding author:llyliliyin@163.com
} 
die. For example, Nagarajan et al [9] earlier researched the fabrication of micro-features on metallic foils using laser-induced shock forming without the assistance of micro-mold patterns. They fabricated micro features with deformation depth of $80 \mu \mathrm{m}$ to $130 \mu \mathrm{m}$ and the radius of $485 \mu \mathrm{m}$ to $616 \mu \mathrm{m}$ was formed on $25 \mu \mathrm{m}$ thick copper foils. Ye et al [10] used fs laser and plasticine to produce micro pits on the pure aluminum foil and investigated the effects of important factors, including pulse widths, impacting times and clamping modes. Zhang et al [11] used plasticine as a flexible support to form micro-craters on the surface of aluminum, cooper, and titanium foils. The results show that mold-free laser shock micro-drawing forming is an effective method to made micro-craters on metallic foils. Dai et al [12] fabricated a micro-dent by a novel net mask shock processing on the surface of LY2 aluminum alloy. Experiments showed that the height of up-warped area around micro-dent was about $4 \mu \mathrm{m}$ and diameter and depth were about $230 \mu \mathrm{m}$ and $9.3 \mu \mathrm{m}$. From the above cases, we can read that almost no one uses mask and plasticine to fabricate micro-features. In the study of forming, plasticine and mask are separately researched, and almost no one uses plasticine and mask to study the forming.

In this article, a new technique named mask and plasticine laser shock forming (MAPLSF) is used to fabricate micro-features on the surface of pure copper foil with the thickness of $0.04 \mathrm{~mm}$. We analyze the profile characteristics of the micro-features and roughness. We also investigate laser energy and expect that the results we get can promote the use of MAPLSF.

\section{Experiments}

\subsection{Forming Mechanism}

Fig.1 shows the principle of the mask and plasticine laser shock forming. The target surface is locally coated with an ablative medium and then covered by a confining medium (such as K9 glass). When the relevant surface of the work piece is irradiated by a high-energy focused and pulsed laser beam, the ablative layer is instantaneously vaporized into a high-temperature and high-pressure plasma. This ablated plasma expands from the work piece surface and, in turn, exerts mechanical pressure on the surface, which would induce compressive waves in the work piece. Plastic deformation of the work piece would be induced by the shock wave.

In this process of MAPLSF, the laser beam and mask are employed as the rigid punch and the micro-mold is replaced by the plasticine which is used as a flexible support. When the laser beams through the mask, it is forced to change into the shape that we need. Relevant ablative layer is irradiated and then vaporized into a high-temperature and high-pressure plasma, forming shock wave. Under the action of shock wave, work piece and plasticine will produce plastic deformation because plasticine is a flexible pad and the bottom of the container has a hole. In this way, we can fabricate the micro-features without the assistant of a rigid micro-mold. 


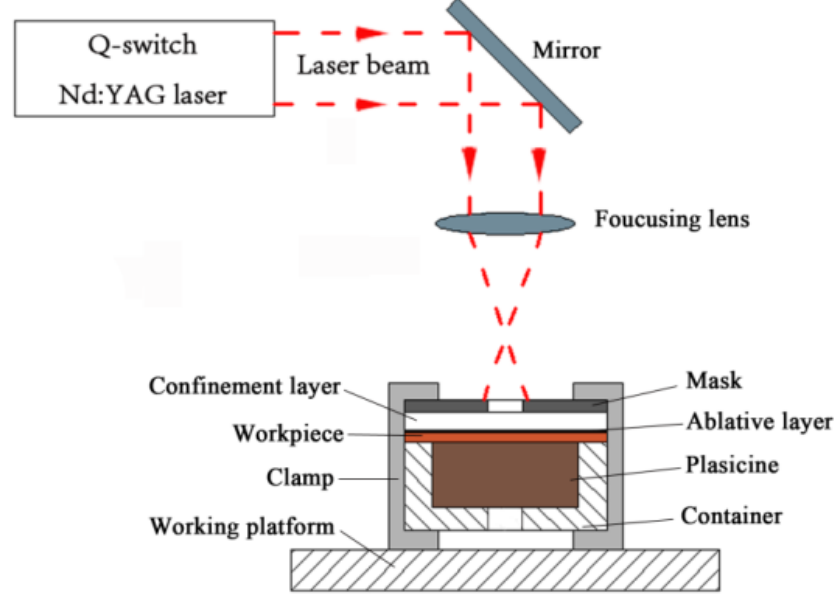

Fig 1. Schematic of laser shock forming

\subsection{Experimental Preparation}

In this experiment, rolled pure copper foil with a thickness of $40 \mu \mathrm{m}$ was used as the work piece. In order to improve the forming performance and decrease the original microstructure defect density, the copper foil was annealed at $450{ }^{\circ} \mathrm{C}$ for an hour and then exposed to furnace cooling in a vacuum. Before performing laser shock, a thin layer of black paint with the thickness of $10 \mu \mathrm{m}$ was sprayed on the copper foil act as an absorbent layer, which can prevent the metal foil from thermal damages. The workpiece and confinement layer are cut into square pieces of $5 \mathrm{~mm} \times 5 \mathrm{~mm}$ and $\mathrm{K} 9$ class serves as the confinement layer. The plasticine was chosen as the flexible support. The flatness of the workpiece and the plasticine should be guaranteed.

For experiments, Spitlight 2000 Nd-YAG Laser with a Gaussian distribution bean is employed. It is operated at the repetition frequency of $10 \mathrm{~Hz}$ and the pulse duration about $8 \mathrm{~ns}$. The wavelength of $1064 \mathrm{~nm}$ is selected. The distance between the mask and the focus must be appropriate in order to ensure that the laser spot is larger than the mask. The mask was cut into rectangular shape with dimension of $50 \mu \mathrm{m} \times 100 \mu \mathrm{m}$ (width $\times$ length ) by wire-electrode cutting, showing in the Fig .2 (a).

The Keyence VHX-1000 digital microscope, Stemi 2000-C microscope and Zeiss CSM 700 true color confocal microscope are employed to examined the three dimensional morphologies on the metal foil surfaces, as well as the ablation state of the ablative medium.

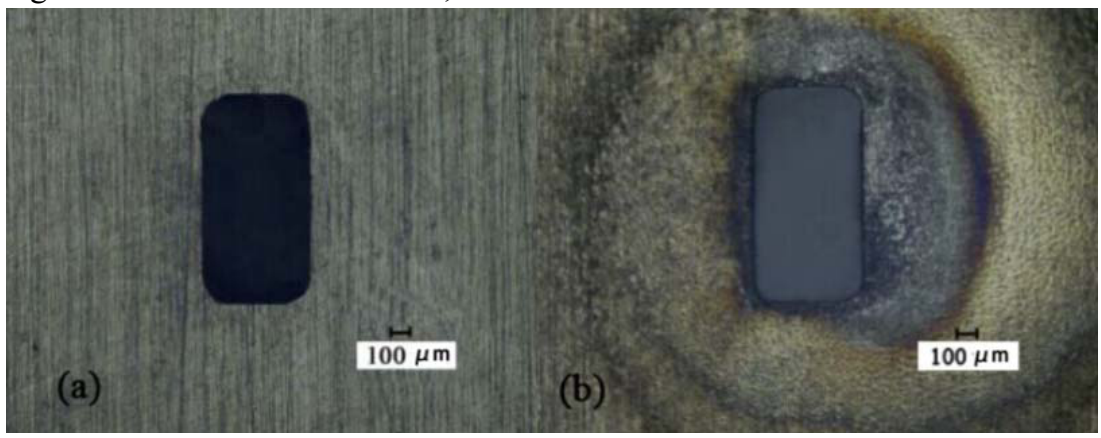

Fig 2 . (a) Without the impact of the mask; (b) After the shock of the mask 


\section{Experimental Results and Discussions}

\subsection{D Micro-topography of the Workpiece}

As can be seen from the Fig .2 (b), laser speckle size is greater than the mask shape and the effect of mask can be seen from Fig 3. Black paint in the region of the direct impact is ablated and the shape is almost the same with the mask. The directly shock area is ablated and other place is not affected. That means mask has a good effect.

Fig .4 (a) presents the topical 3D profiles of the micro-feature. From the picture, we can see that the depth of micro-feature is about $353.8 \mu \mathrm{m}$ with the maximum deformation depth at the center of the workpiece and forming surface is very smooth, no deformity or defect part. The phenomenon could be attributed to the two factors: (1) The hardness of plasticine is lower than that of workpiece, so the formation of the surface is smooth; (2) In the process of forming, plasticine impacted by the shock wave, generates plastic deformation and excess plasticine goes through hole to the blow container at the same time. Therefore the workpiece surface does not have any spring-back. We can clearly see from Fig .4, in the bottom of the workpiece, there is a clear similar to the shape of the mask. But the shape in the rectangular box is smaller than directly shock area.

\subsection{Effect of the Laser Energy}

Laser energy is one of the key parameters in the process of MAPLSF, therefore, it is necessary to conduct experiment to analyze the influence of the laser energy on the deformation of thin metal foils. The energy is controlled by the percentage, $50 \%, 60 \%, 70 \%$, $80 \%, 90 \%$, and $100 \%$,corresponding $1020,1200,1380,1550,1690$, and $1900 \mathrm{~mJ}$, respectively.

Fig .5 is a typical central profile line of the micro-feature. It is found that the diameter of the micro-feature is much bigger than the mask size after shock process. So, the total forming depth is composed of two parts. One is the directly forming depth, which is located at the central of the micro-feature and the other part is indirect forming depth. The two regions will connect smoothly, as seen in Fig .5. The experiment was repeated three times, and the average values were adopted to draw in Fig .6.

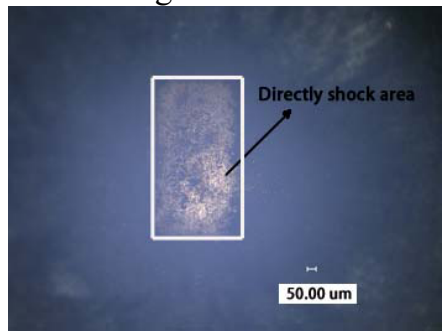

Fig 3. The shape of the ablation 


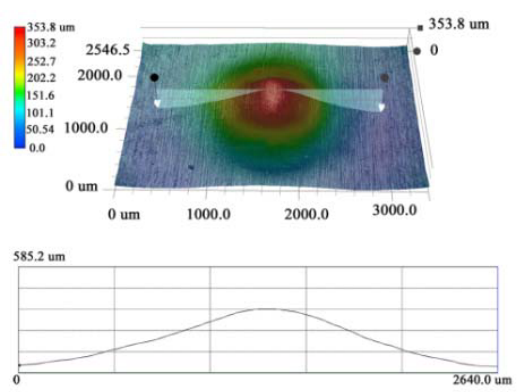

(a)

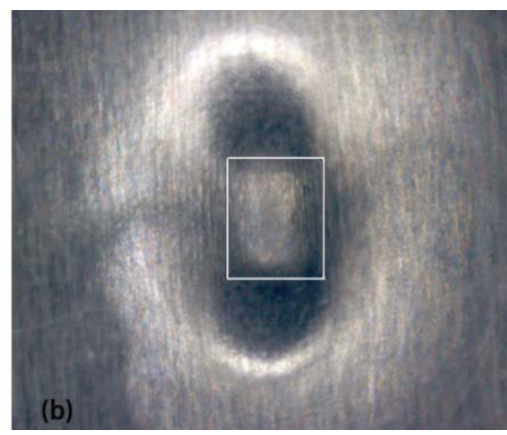

Fig 4. (a) The magnification micro-topography and surface profile of the micro-feature; (b) The top view of the

It is observed that the increase of the total forming depth can be explained by the increase of laser energy. From the Fig .6, depth is always growing. Several reasons can be explained this phenomenon: (1) Black paint is not fully be ablation, so with the increase of laser energy, the blank paint constantly absorbs energy, producing large amounts of plasma, and the pressure increases at the same time. Therefore, when the black paint is ablation completely, the forming depth would be reduced; (2) Plasticine has good ability of plastic forming, and does not produce spring back in the forming process. So the total forming depth and the directly forming depth are always increasing, when energy within $1900 \mathrm{~mJ}$.

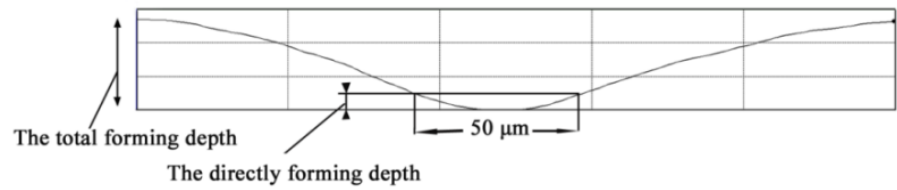

Fig 5. The forming depth of micro-feature

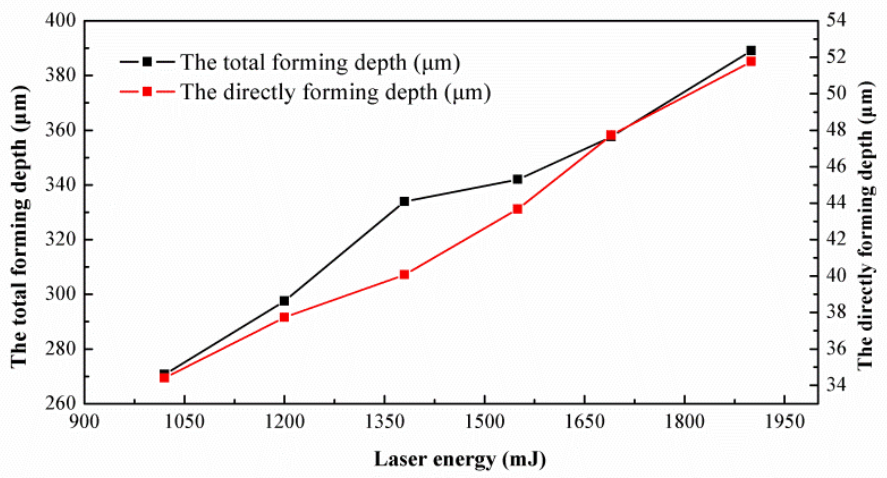

Fig 6. The total and directly forming depth of the micro-feature

\subsection{The Roughness of the Work Piece}

As we mentioned before in this paper, the roughness of the workpiece would increase when we used the rigid mold to form. The mold is very difficult to be manufactured, and the roughness is more difficult to be guaranteed. Luo et al [13] have done the relevant research and the result is displayed in Fig .7. The process has shown a better replication ability for 
local micro structures with $20-50 \mu \mathrm{m}$ in width and $1-7 \mu \mathrm{m}$ in height, as their replication degree could reach $84 \%$. Therefore, the roughness of the workpiece will increase.

In this process of MAPLSF, the hardness of the plasticine is smaller than the workpiece, so the surface of the workpicec does not have damage. Fig .8 shows the morphology of the raw material and the forming material. It can be seen clearly from the Fig .8 and the forming surface has no obvious highlight or depression. Black paint can protect the up surface from the effects of laser ablation, and the down surface is support by the plasticine, which is a flexible-pad. So the workpiece can have a good surface after the process of laser shock forming.
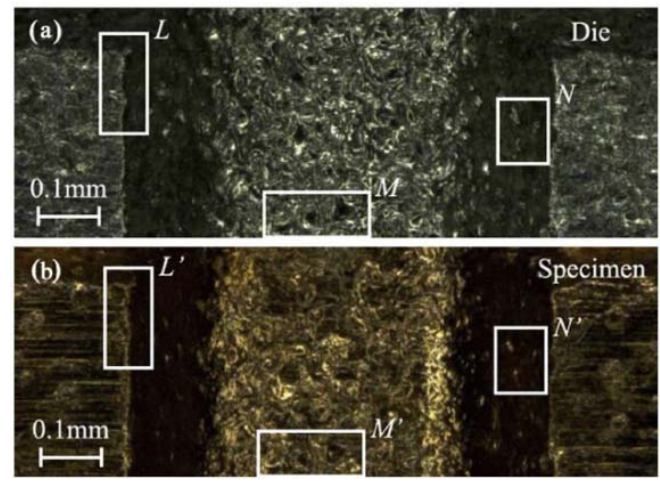

Fig 7. (a)Enlarged photo of the local area of the die;

(b)Enlarged photo of a specimen in the local area corresponding to (a).

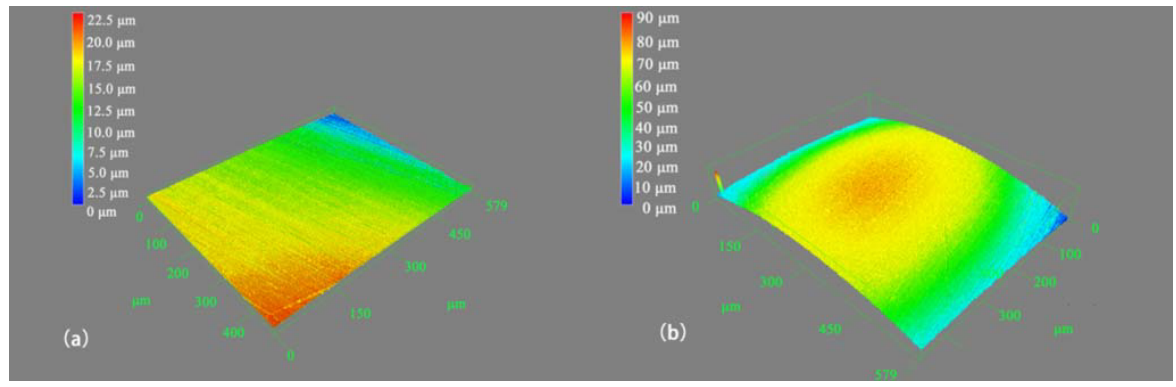

Fig 8. (a)The morphology of raw material; (b)The morphology of forming material

\section{Conclusions}

This article presents a new type of laser shock forming, named MAPLSF, which can deform different micro-features in the surface of the workface. Some important findings of this work are listed as follows:

(1)From the result of the experiment, we conclude that the shape of laser beam can be changed very well by the mask. The shape of light beam can be roughly manufactured on the surface of the workpiece, when we use the mask and plasticine in the forming process.

(2)It is found that the laser energy is an important parameter in the process of the MAPLSF. With the increasing laser energy, the depth of the micro-feature increases, and the depth reaches a limiting value at a high energy.

(3)The workpiece can have a good surface when we use the plasticine as a flexible-pad, which have the lower hardness. Therefore the surface of the workpiece will not be damaged. 


\section{Acknowledgments}

This work is supported by the National Natural Science Foundation of China (No.51175235), the Natural Science Foundation of Jiangsu province (No. BK20151343.), the scientific research project of Jiangsu University (No.14A120) and the college students practice innovation fund of Industrial Centre of Jiangsu University (No. ZXJG201590).

\section{References}

1. F. Vollertsen, H.S. Niehoff, Z. Hu: International Journal of Machine Tools and Manufacture [J], Vol. 46 (2006), pp: 1172-1179.

2. R. Fabbro, P. Peyre, L. Berthe, et al: Journal of laser applications [J], Vol,10(1998), pp: 265-279.

3. D. Wang, T. Shi, J. Pan, et al: Journal of Materials Processing Technology [J], Vol. 210(2010), pp: 684-688.

4. M.W. Fu, B. Yang, W.L. Chan: Journal of Materials Processing Technology [J], Vol. 213(2013), pp: 101-110.

5. H. Zein, M.E. Sherbiny, M. Abd-Rabou, et al: Materials \& Design [J], Vol. 53(2014), pp: 797-808.

6. M. Zhou, Y.K. Zhang, L. Cai: Applied Physics A [J], Vol. 77(2003), pp: 549-554.

7. F. Vollertsen, H.S. Niehoff, H. Wielage: Production Engineering [J], Vol.3( 2009), pp: $1-8$.

8. H. Gao, G.J. Cheng: Microelectromechanical Systems [J], Vol. (19)2010, pp 273-281.

9. B. Nagarajan, S. Castagne, Z. Wang, et al: Applied Surface Science [J], Vol, 268(2013), pp: 529-534.

10. Y. Ye, Y. Feng, Z. Lian, et al: Optics and Lasers in Engineering [J], Vol, 67(2015), pp: 74-82.

11. D. Zhang, Y. Lin, C. Gu, et al: The International Journal of Advanced Manufacturing Technology [J], Vol,79(2015), pp: 265-272.

12. F.Z. Dai,J.Z. Lu, Y.K. Zhang, et al: Journal of applied physics [J], Vol,112(2012), pp: 023117.

13. F. Luo , K. Li, J. Zhong, et al: Journal of Materials Processing Technology [J], Vol,216(2015), pp: 10-18. 